
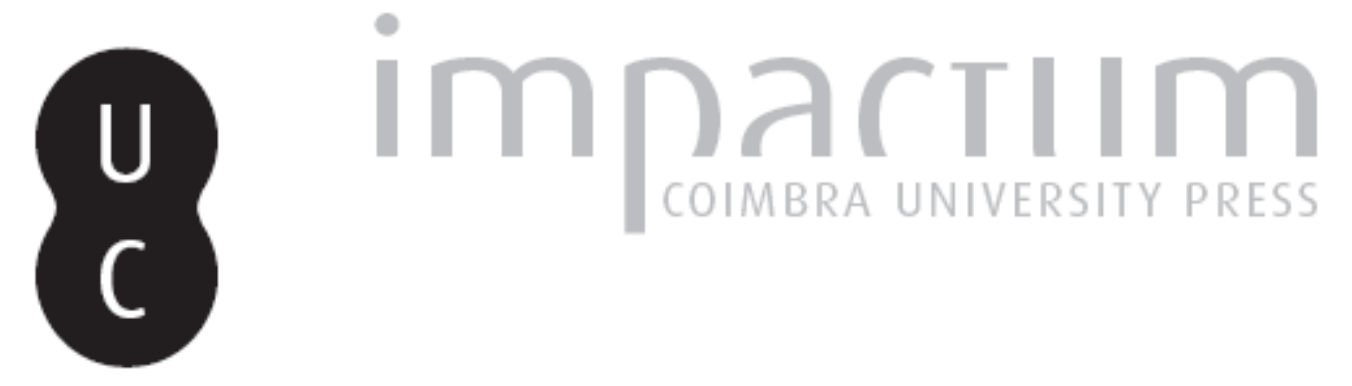

\title{
L'estetica del comico e la Aristophanis et Menandri comparat
}

\section{Autor(es): $\quad$ Fiorio, Maria Di}

Publicado por: International Plutarch Society

URL persistente:

URI:http://hdl.handle.net/10316.2/37572

DOI:

DOI:http://dx.doi.org/10.14195/0258-655X_1_2

Accessed : $\quad$ 26-Apr-2023 10:21:49

A navegação consulta e descarregamento dos títulos inseridos nas Bibliotecas Digitais UC Digitalis, UC Pombalina e UC Impactum, pressupõem a aceitação plena e sem reservas dos Termos e Condições de Uso destas Bibliotecas Digitais, disponíveis em https://digitalis.uc.pt/pt-pt/termos.

Conforme exposto nos referidos Termos e Condições de Uso, o descarregamento de títulos de acesso restrito requer uma licença válida de autorização devendo o utilizador aceder ao(s) documento(s) a partir de um endereço de IP da instituição detentora da supramencionada licença.

Ao utilizador é apenas permitido o descarregamento para uso pessoal, pelo que o emprego do(s) título(s) descarregado(s) para outro fim, designadamente comercial, carece de autorização do respetivo autor ou editor da obra.

Na medida em que todas as obras da UC Digitalis se encontram protegidas pelo Código do Direito de Autor e Direitos Conexos e demais legislação aplicável, toda a cópia, parcial ou total, deste documento, nos casos em que é legalmente admitida, deverá conter ou fazer-se acompanhar por este aviso.

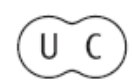




\title{
L'ESTETICA DEL COMICO E LA Aristophanis et MENANDRi CoMparatio da Maria Di Florio Università di Salerno
}

\begin{abstract}
Some literary essays are listed from No.119 to No.123 in the Catalogue of Lamprias. In particular, there is a lost treatise of literary criticism -whose we have a compendium - on a comparison between Aristophanes and Menander, in which Plutarch praises the poet of the New Comedy (No.121: Aristophanis et Menadri Comparatio). This work wants to search into the cause of the writer's preference and it examines his ideas of 'vis comica'. At first, Plutarch points out the style, then he

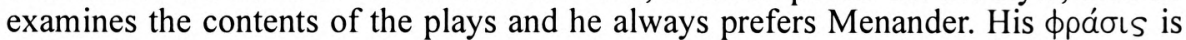
polished and suits many characters in spite of Aristophanes, who stuffed his plays with too many figures of speech. Moreover, Menander is right on every occasion, at school, at theatre, at symposium, whereas Aristophanes likes to show on the stage arrogance, intemperance and malice and he is unpleasant. Anyway, beyond the plays on words and the vulgarity, Plutarch seems to blame the wit of Aristophanes, because it is unprejudiced and useless; Menander, on the contrary, has a very kind sense of humour, and in his plays the life of the times is reflected somewhat.

So, Plutarch's judgement reflects a careful theory. To make good comedies it is

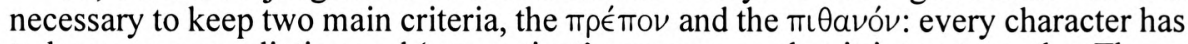
to have a proper diction and 'convenient' manners, so that it is trust-worthy. Therefore, Plutarch rejects Aristophanes because he shows unlikely or improbable events and everything he 'imitates' is worse than it is. Also Menander's language is better and it doesn't carry its Atticism too far.

Finally, Menander's plays are very usefull to educate young people and they have an ethic power, which sometimes is almost cathartic.
\end{abstract}

Nella copiosa varietà tematica che presentano gli scritti del corpus dei Moralia di Plutarco di Cheronea risultano annoverati anche saggi letterari, alcuni dei quali si trovano raggruppati tra il numero $119 \mathrm{e}$ il numero 123 del Catalogo di Lampria.

Dedicati ad Arato (119), a Nicandro (120), ad Aristofane e Menandro (121), ad Erodoto (122) e all' Iliade (123), essi costituiscono una riprova della vasta erudizione e della sicura competenza che il Cheronese poteva vantare nel campo della letteratura greca.

Senza addentrarci ora nelle oscure questioni sul metodo storiografico di Erodoto e prescindendo pure dagli altri saggi, di cui rimangono appena i titoli, concentreremo la nostra attenzione su quell'opuscolo che figura proprio al centro di detto gruppo e che fornisce uno spaccato molto interessante della critica 
letteraria, in particolare legata all'arte drammatica, di età imperiale.

La Aristophanis et Menandri Comparatio, per noi perduta, di cui tuttavia un anonimo compendium ha conservato fedele memoria, produce una valutazione in forma comparativa dei due più insigni rappresentanti del teatro comico greco e si risolve in una convinta esaltazione del comico della véa a dispetto del grande poeta dell'à $\rho x \alpha i ́ \alpha$.

Partito da una considerazione di natura stilistica, che vede contrapposte l'espressione ridondante e infarcita di artifici retorici, spesso non appropriata, tipica di Aristofane (853B) e quella levigata e tersa di Menandro, adatta, invece, ad esprimere tutte le emozioni e a confarsi a diversi personaggi, uniforme pur nella sua eterogeneità e nonostante qualche isolato effetto reboante qualora l'azione lo richieda (853D), Plutarco non ha dubbi su cosa scegliere e, intanto, inserisce nell'argomentazione ben otto citazioni aristofanee con valore esemplificativo, per denunciare, con maggiore incisività, il cattivo gusto del suo bersaglio polemico (853C-D). Passa, poi, su un piano più chiaramente contenutistico e mentre ad Aristofane imputa arroganza, intemperanza e malizia, tutte pessime qualità che lo rendono sgradito e intollerabile sia alle masse sia ai saggi, quasi simile ad un'etera sfiorita che volesse far la moglie (854A), di Menandro, al contrario, apprezza la "grazia" che lo contraddistingue in ogni occasione, nei teatri nelle diatribe nei simposi, e riconosce come soprattutto per gli intellettuali egli sia ristoro dai loro studi concentrati e intensi, sortendo lo stesso effetto ricreante di un bel prato fiorito, ombroso e pieno di brezze (854B-C). In chiusura, infine, come a ribadire l'accusa nella pronuncia del verdetto, sferra l'attacco definitivo e, dopo aver attribuito ad Aristofane sales di un'acerbità esulcerante e mordace, dice che egli rende peggiore di quello che è qualsiasi cosa rappresenti, per cui l'astuzia non è urbana ma maligna, la rustichezza non è semplice ma sciocca, il ridicolo non è scherzoso ma risibile, e l'amore non è lieto ma lascivo, sì da riscontrare un buon successo solo tra i libertini e i maligni, non di certo tra gli uomini morigerati (854C-D).

Al di là dell'artificio stilistico e della volgarità dei contenuti, però, quello che Plutarco sembra deplorare più di ogni altra cosa in Aristofane è il prevalere di una vis comica spregiudicata e fine a se stessa, improntata all'immaginoso e al paradossale, assai lontana da quel senso umoristico di Menandro insieme "fine ed elegante", che tanto piace agli amanti della $\mu \varepsilon \sigma o ́ t \eta S^{1}$. Alla luce di questa

1 Cf. M. R. CAmmarota, "Estetica e critica letteraria in Plutarco", Quaderni del Dipartimento di Scienze dell'Antichità - Università di Salerno, 6 (1990) 91-108, in part. p. 105; L. VAN DER STOCKT, Twinkling and Twilight, Plutarch's Reflections on literature, Brussel, 1992, pp. $160 \mathrm{~s}$. 
conclusione, allora, si comprende come l'opuscolo non si limiti ad esibire una semplice analisi letteraria, ma lasci intravedere delle idee ben precise, che possono riconoscersi come peculiari del Cheronese e stimolano una riflessione più generale sulla sua estetica del 'comico'.

Va premesso, a tal proposito, che non vi è nell'intera produzione plutarchea una sistematica definizione della sua teoria del comico, per cui bisognerà dedurla raccogliendo, esaminando e confrontando gli spunti occasionali disseminati qua e là. $\mathrm{Ci}$ introdurrà, comunque, a tale lavoro l'osservazione della consueta modalità di approccio alla categoria mentale del comico e il vaglio del contenuto semantico con cui sono usati i termini che afferiscono a tale sfera. Si nota che a differenza di quello che accade per il tragico ${ }^{2}$, Plutarco non è solito applicare un concetto generico di comico, che esuli dal campo letterario e sia percepito, secondo il moderno uso comune, alla stregua di un'esperienza di vita. Mentre molto spesso, infatti, nelle opere del Cheronese rileviamo che l'accezione più corrente in cui sono intese le parole 'tragico' e 'tragedia' è quella di "avvenimento grandioso e sconvolgente [...] con risonanze particolari su determinati stati d'animo", , le parole 'comico' e 'commedia', invece, non risultano usate, come noi ci aspetteremmo, per indicare un fatto a lieto fine e magari 'condito' di battute divertenti e tante risate. In definitiva, per

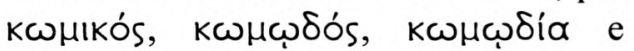
$\kappa \omega \mu \omega \delta \varepsilon \varepsilon^{\prime} \omega$ non si registrano sfumature connotative di significato, ma il loro impiego appare legato in modo quasi assoluto alla rappresentazione drammati$\mathrm{ca}^{4}$. La sola precisazione che si può fare è che la prospettiva del riferimento cambia spostandosi alternativamente dal genere letterario, cui si dedicano i poeti ${ }^{5}$, alla in part. pp. 125 ss.; L. Di Gregorio, "Plutarco e la tragedia greca", Prometheus, 2 (1976) 151-174, in part. pp.169 ss.

A. M. TAgliasacCHI, "Plutarco e la tragedia...", cit., p. 125.

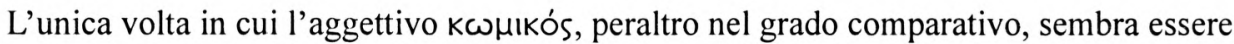
utilizzato in senso diverso è in Galb. 12,3 dove è riferito alla $\mu \varepsilon т \rho$ то́тпs di Cesare nei confronti di Vinio.

Cf. Alc. 10,4; 13,4; 13,9; 20, 6; Alex. 17,7; 29,6; Arist. 5,8; Cim. 10,4; Dem. 9,4; 9,5; Demetr. 12,6; 12,7; 27,4; Luc. 39,1; Lys. 13,5; Nic. 4,5; 11,6; Num. 8,9; Per. 3,5; 4,4; 7,8; 8,4; 13,15; 16,1; 24,9; 32,1; Pomp. 53,6; Sol. 25,2; T.G. 30,2; Them. 19,4; 32,6; adulat. 55C, 59C, 62E, 66D, 68B; aet. Rom. 288A; amat. 750F; an seni resp. 785B; apopht. 175C, 183E; apopht. Lac. 239B; col. 1127A; comm. not. 1065D-E, 1066A; cons. ad Apoll. 105F, 110E; curios. 517B, 518A; def. orac. 420D; exil. 602B; garr. 508C, 513E; glor. Ath. 347E, 348B; Herod. mal. 855F; praec. ger. reip. 801B, 807A; Pyth. or. 395D; quaest. conv. 615D, 631D, 634D, $666 \mathrm{E}, 710 \mathrm{C}, 711 \mathrm{~F}, 712 \mathrm{~B}, 739 \mathrm{~F}$; superst. $166 \mathrm{~B}$; tuend. san. $126 \mathrm{~A}$; $X$ orat. vit. $839 \mathrm{~F}$. 
vera e propria messa in scena da parte degli attori ${ }^{6}$.

Se, dunque, per Plutarco l'essenza del comico converge con l'essenza della commedia, si comprende l'importanza che riveste la Comparatio. A ben guardare, infatti, essa presenta tutta una serie di elementi che meritano di essere presi in considerazione tanto più seriamente, in quanto rivelano le stesse frequentazioni filosofiche del Cheronese. Così, la preferenza accordata a Menandro, che emerge con evidenza dal testo, oltre a coincidere con l'esito di un mero giudizio di critica letteraria, è indicativa di un effettivo orientamento teorico ${ }^{7}$.

Il primo criterio fondamentale ad essere introdotto, pur in termini negativi per Aristofane, è quello del 'conveniente' (тò тре́тоv), che Plutarco applica qui precisamente allo stile $(853 \mathrm{D}: \dot{\eta}$

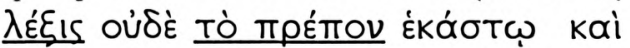

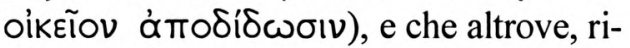
ferito a tutta quanta la poesia, di qualsiasi genere letterario, sembra identificarsi per lui con la definizione stessa del 'bello'.

La critica allo stile di Aristofane, infatti, è innanzi tutto diretta contro l'impiego 'spropositato' di figure retoriche e giochi di parole privi di alcun senso, ma, poi, ancor di più, uniformandosi apertamente alla scuola peripatetica, essa si appunta sull'incapacità del commediografo di assegnare a ciascun personaggio ciò che gli si conviene?

Cf. Ant.29,4; 70,2; Cic. 5,4; Cim. 16,8; Comp. Dem. et Cic. 1,5; Dem. 4,6; Demetr. 28,1; Nic. 4,6; Per. 13,8; Publ. 20,4; Sull. 36,1; adulat. 50E; Alex fort. virt. 334E; amat. 769D; am. prol. 497B; an seni resp. 789C; aud. poet. 27C; coh. ira 458A; col. 1120D; cum princ. philos. 778E; curios. 517A, 519B, 521E; def. orac. 431C; exil. 600B; laud. ips. 545E; lib. ed. 10C; quaest. conv. 665E, 673C, 676C, 712A; tranq. an. 473B; vit. pud. 531B; $X$ orat. vit. $833 \mathrm{C}, 836 \mathrm{~F}, 841 \mathrm{~F}, 845 \mathrm{~B}$.

Cf. A. PleBe, La teoria del comico da Aristotele a Plutarco, Torino, 1952, pp. $104 \mathrm{~s}$. Riflettendo sulla fortuna postuma di Menandro, in un saggio a lui dedicato, D. LANZA ("Menandro", in Lo spazio letterario della Grecia antica, I, 2, Roma, 1993, pp. 501-520, in part. p. 502) si sofferma proprio su questo opuscolo e dice che "la comparazione plutarchea è tra due autori, ma si può facilmente immaginare come dietro i nomi dei due poeti siano qui presentati anche due diversi modi di scrivere commedie, forse addirittura di intendere la stessa essenza del comico".

Cf. Plu., aud. poet. 18A-D, dove lo scrittore più che sull' 'adeguamento' stilistico, insiste sulla fedeltà di riproduzione di ogni soggetto, secondo quelle che sono le sue caratteristiche peculiari. A tal proposito, egli puntualizza che un'imitazione fatta "in modo bello" signifi-

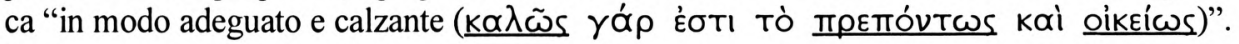

Nella centralità della 'convenienza' stilistica nella poesia comica si può avvertire, in particolare, più che quella di Aristotele l'influenza di Teofrasto che aveva schematizzato l'elocuzione tipica della commedia come una 'pittura di caratteri', perfettamente inquadrata, peraltro, nella sua rigorosa sistemazione dei generi letterari. Cf. A. PLEBE, op. cit., pp. 23s. e pp. 41 ss. 
Perché ogni opera poetica, e la commedia in particolare, risulti 'bella' agli occhi di Plutarco, occorre, dunque, che alla descrizione di ognuno corrisponda un peculiare tratto stilistico oltre che un tipo di eloquio che si addica alla sua condizione: la gravità al re, l'eloquenza al retore, la semplicità alla donna, la prosaicità all'uomo di mercato, in modo tale, insomma, da ottenere "i detti turpi in bocca alle persone dappoco e i detti buoni in bocca a personaggi decorosi" 10 .

Strettamente collegato col primo è il concetto del 'verosimile' (Tò miӨ Infatti, se il criterio della convenienza funziona e i discorsi sono appropriati alla realtà dei personaggi, apparirà chiaro dalla 'verosimiglianza', ovvero dalla 'persuasività' dell'opera stessa ${ }^{11}$. Non a caso, è questo un motivo di preferenza per le commedie di Menandro, visto che egli "si insinua dappertutto, offrendo un grado di persuasione che non ammette

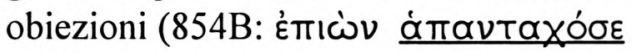

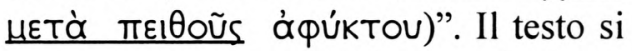
presta ad una duplice interpretazione: da una parte indica la prerogativa dell'opera menandrea di rivelarsi appropriata in ogni ambiente e, quindi, in diverse occasioni -appena qualche riga prima si è detto che egli risulta soddisfacente a scuola, a teatro e nei simposi- ma al tempo stesso allude anche al fatto che egli sa trattare ogni tipo di argomento adeguatamente $^{12}$. La persuasività, in tal caso, sarà tanto quella dei discorsi, quanto quella delle azioni dei personaggi. Cominciamo, pertanto, a capire che è solo attraverso una 'convenienza' di ampio raggio e per effetto di una 'verosimiglianza' riguardante tanto lo stile quanto il contenuto ${ }^{13}$, che il poeta riesce

Ibidem, p. 102.

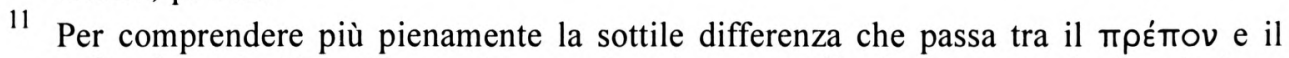
$\pi \mid \theta \alpha \nu$ òv e che li rende complementari, può essere utile riproporre anche in relazione allo stile quello che E. VALGiglio ("Il tema della poesia nel pensiero di Plutarco", Maia, 19 (1967) 319-355, in part. p. 322 n. 10) dice a proposito della mimesi: "il $\pi \bullet a \operatorname{còv}[\ldots]$ sembra in ragione dell'imitazione in senso passivo, dell' imitato, in quan-

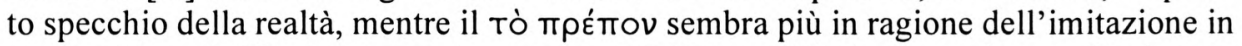
senso attivo, dell'imitante, quale canone artistico; se l'imitazione rispetta l'esigenza

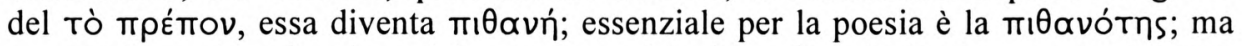

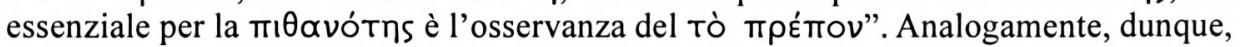

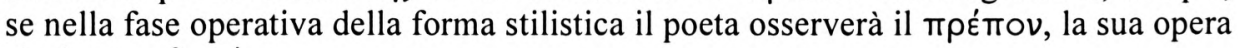
risulterà mı $\theta$ avn.

In questo modo intendono propriamente sia Fowlen (Plutarch's Moralia, vol. X, London-Cambridge-Massachussets, 1936), il quale traduce: "approaching all subjects with a persuasiveness from which there is no escare", sia LACHENAUD (Plutarque. Oeuvres Morales, t. XII-1, Paris, 1981), il quale traduce: "qui parcourt tous les sujets avec une force de persuasion invincibile". Cf. anche L. VAN DER STOCKT, op. cit., p. 159.

Per la verosimiglianza degli argomenti in Teofrasto, cf. A. PLEBE, op. cit., p. 23 e pp. 35 ss. 
a veicolare credibilmente il suo messaggio, sì da poter esercitare favorevolmente una sorta di funzione 'psicagogica' 14 .

Ancor più del canone stilistico, anzi, è importante 1' 'argomento', il fatto di cui si tratta nella commedia. Una conferma si può riscontrare facendo un passo indietro, all'inizio del secondo capitolo dell'opuscolo, là dove si precisa come

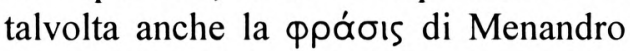
conosce un effetto prodigioso, qualora $\mathrm{l}^{\prime}$ 'azione' lo richieda (853E: żàv $\delta \varepsilon ́$ TI-

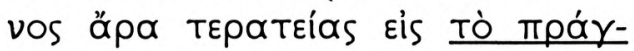

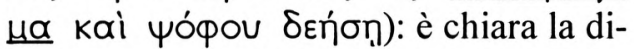
pendenza e, quindi, la subordinazione dell'espressione formale rispetto all'in- treccio. Questo spunto, inoltre, non rimane un caso isolato, ma è ripetuto più volte, rivelandosi una ferma convinzione del Cheronese, originata quasi sicuramente dal modello aristotelico ${ }^{15}$. Ł̀ sintomatico, ad esempio, quanto è detto in aud. poet. 16B, dove lo scrittore sottolinea la preminenza dell'intreccio su tutti gli artifici metrici e retorici: "non esistono metro, figure stilistiche, turgore espressivo, felicità di metafore o armonia compositiva, che siano in grado di esercitare una suggestione e un fascino paragonabili a quelli di una composizione mirabilmente intrecciata

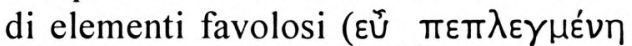

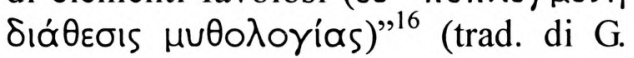

14

Ma ancora moltissimi sono i passi plutarchei in cui si preferisce un buon concetto all'orpello esteriore: cfr. Plu., garr. 510E e Lyc. 19 (dove si loda Licurgo, che insegnò ai giovani spartani ad esprimersi con poche e concise parole); aud. 42D-E (dove, indirettamente, il contenuto è paragonato prima ad un utile antidoto, mentre lo stile al materiale di cui è fatto il recipiente, poi ad un caldo mantello di lana, mentre lo stile alla qualità della lana stessa); prof. virt. 79C (dove si consiglia di non maneggiare le poesie per semplice diletto e gioco, bensì, piuttosto, per cercarvi preziosi concetti). In qualche caso, poi, tale preferenza dà origine ad una vera e propria 'accusa' della forma, come in aud. poet. 28D-E (dove si insiste sull'utilità della poesia nonostante i suoi elementi di puro ornamento) o in Pyth. or. 397A-B (dove a proposito della Sibilla si insiste sulla 'essenzialità' e sull'assoluta 'purezza' degli oracoli) oppure, ancora, in coniug. praec. 142A-B (dove un buon contenuto è consigliato contro una cattiva forma). Altre volte, viceversa, Plutarco sa riconoscere un certo valore anche alla forma sicché in quaest. conv. 747E-F considera l'importanza del verso aggraziato e melodioso, mentre in quaest. conv. 623 A

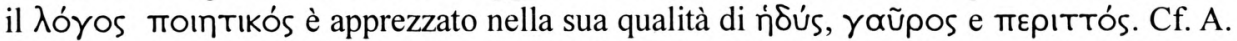


Pisani) ${ }^{17}$. Per la commedia, in particolare, lo stesso concetto torna con maggiore evidenza in glor. Ath. 347E-F, a proposito di un aneddoto su Menandro: un giorno, poiché erano giunte le feste Dionisiache e il poeta non aveva ancora composto la commedia da presentare al concorso, un amico gliene chiese preoccupato la ragione ed egli rispose che in realtà la commedia poteva ritenersi pronta, perché ne era già organizzata la

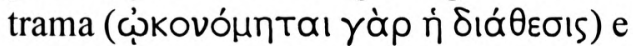
bisognava solo metterla in versi $(\delta \varepsilon \tilde{i}$

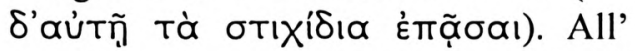
aneddoto segue poi il commento di Plutarco che nota come i poeti stessi comprendessero che nella poesia i fatti sono più 'necessari' e 'importanti' delle

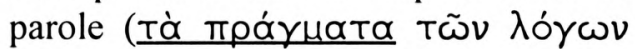

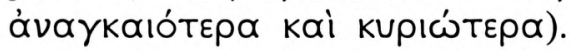

Sia dal primo che dal secondo passo emergono indizi di quella che è la materia che si offre come argomento per la poesia in genere e, nel caso specifico, per la poesia comica. Nel primo, in particola- re, si parla di una "composizione mirabilmente intrecciata di elementi favolosi", mentre nel secondo si allude ad una trama che il poeta avrebbe già in mente: ne deduciamo, pertanto, che il contenuto della poesia è qualcosa di 'inventato'.

L' 'invenzione', il 'falso' $o$, in altre parole, le 'menzogne' dei poeti ${ }^{18}$, coincidono con quell'essenza mitologica che è parte costitutiva della poesia stessa, secondo una convinzione largamente diffusa nell'antichità greca ${ }^{19}$, e che è dotata di un fascino pericoloso per il giovane, perché fuorviante.

Era questa la principale imputazione della famosa condanna che Platone formulava contro la poesia, eliminandola dalla sua Repubblica ideale ${ }^{20}$. Eppure, nonostante sia un seguace fedele del 'divino' filosofo, come esplicitamente lo chiama $^{21}$, Plutarco supera il condizionamento del Maestro e 'salva' la poesia accettandone la falsità, in virtù di quella stessa funzione etico-pedagogica dell'arte che vedremo avere un peso note-

M. TAGLiasacchi, "Le teorie estetiche...", cit., pp. 99 ss. ; E. VAlgiglio, "Il tema della poesia...", cit., p. 320; M. R. Cammarota, "Estetica e critica letteraria ...", cit., pp. 99 ss. Plutarco, Moralia, II. L'educazione dei ragazzi, a c. di G. PISANI, Pordenone, 1990. 20; S. E., M. 1.297; D. Chr., or. 11.7. Per le testimonianze sulla paternità teofrastea del concetto di invenzione comica cf. A. PLEBE, op. cit., pp. 32 ss. 
vole nella sua concezione estetica ${ }^{22}$.

E' vero anche, però, che si riconoscono delle esplicite restrizioni da parte di Plutarco: egli non ama il 'falso' puro, scevro da qualsiasi rapporto con la realtà, e che andrebbe solo a generare confusione $\mathrm{e}$ distrazione. Piuttosto, come affema in aud. poet. $16 \mathrm{~B}$, predilige un $\mu \varepsilon \mu 1 \gamma \mu \varepsilon ́ v o v$

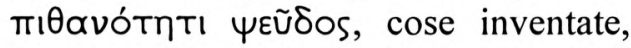
insomma, ma che somiglino alla realtà. Si capisce perfettamente, allora, come il Cheronese non riuscisse ad accettare la commedia di Aristofane e l'ópxaía in genere, dal momento che essa metteva sulla scena esperienze spesso 'irreali' e 'irrazionali', e come, invece, trovasse piena soddisfazione in quella commedia nuova tutta tesa a rappresentare la vita ${ }^{23}$.

La constatazione che il falso è vincolato, per lui, al 'verosimile' spinge la nostra riflessione a spostarsi su un nuovo punto dell'estetica plutarchea. Intrecciato al motivo dell'invenzione appare, infatti, un altro elemento che viene introdotto verso la fine della Comparatio, dove Plutarco dice di Aristofane che qualunque cosa egli imiti la volge in

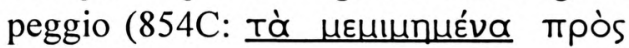

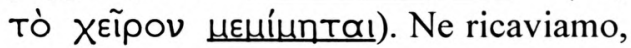
pertanto, un'ennesima prova -questa volta relativa alla poesia comica- che essenza dell'arte poetica è la mimesi, secondo un'idea variamente presente alla tradizione precedente ${ }^{24}$. Lo spunto

G. D'IPpolito ("Politica e poetica in Plutarco", in Teoria e prassi nelle opere di Plutarco, Atti del V Convegno plutarcheo (Certosa di Pontignano, 7-9 giugno 1993) a c. di I. Gallo e B. Scarmigli, Napoli, 1995, pp. 121-134, in part. pp. 128 ss.) rileggendo i cosiddetti capitoli 'teorici' del De audiendis poetis (2, 3 e 7), li interpreta come una manifesta risposta alla condanna platonica, constatando come l'impostazione del discorso segua a tutti gli effetti le linee direttrici dei capi d'accusa platonici, al fine di smontarli uno per uno. D'altra parte, invece, C. BRECHET ("Le audiendis poetis de Plutarque et le procès platonicien de la poésie", $R P h, 73$ (1999) 209-244, in part. pp. 210 ss.) sottolinea come in realtà il Cheronese affrontasse il problema su un piano completamente diverso da quello di Platone, perché secondo una linea di pensiero storicamente diversa, sia per condizione politica sia per importanza della filosofia. Pertanto la posizione di Plutarco, a suo avviso, non dovrebbe essere considerata 'difensiva' della poesia, ma semplicemente diversa: "il ne s'agit pas de répondre terme à terme aux critiques formulées par Platon dans la République, mais de montrer comment on peut d'une part conjurer la nocività des poèmes, et d'autre part tirer d'eux ce qu'ils contiennent de positif' (p. 212). Cf. D. LANZA, "Lo spettacolo", in AA.VV. Oralità scrittura spettacolo, a c. di M. VEGETTI, Torino, 1983, pp. 107-126, in part. pp. 119 ss.; M. R. CAMMAROTA, "Estetica e critica letteraria...", cit., pp. $106 \mathrm{~s}$.

24 Se per Platone, ad esempio, la mimesi artistica assumeva un valore negativo, dal momento che l'opera d'arte in quanto imitazione della realtà effettiva e contingente, a sua volta immagine imperfetta dell'Idea, era lontana tre gradi dal vero (Cf. Pl., R. X 597e), per Aristotele, invece, la mimesi non coincideva più con la copia del reale, ma tendeva alla forma ideale col puro sforzo dell'immaginazione (cf. Arist., Po. 1460 b 9-10; 1451 a 37). 
appare ben sviluppato ancora una volta nell'opuscolo pedagogico del $D e$ audiendis poetis, dove peraltro viene ripreso un parallelo di reminiscenza sia platonica che aristotelica ${ }^{25}$, per cui la mimesi poetica è accostata alla mimesi della pittura: la poesia è una pittura che parla e la pittura una poesia che tace ${ }^{26}$.

Certo, però, per quanto attiene alla commedia il motivo mimetico si complica.

Se la commedia, infatti, come la definì Aristotele è $\mu$ íf essa comporta la rappresentazione di azioni 'cattive' e spettacoli 'brutti' (i due principi di valutazione sono assolutamente collegati per Plutarco), che non possono non turbare un moralista pedagogo dello stampo del Nostro. Allora, come risolvere questa aporia?

Il Cheronese affronta il problema in contesti diversi, ma è chiaro che la soluzione proposta vale anche per il teatro comico: l'unica risorsa per cui le azioni indegne e i personaggi indecorosi (anche della commedia) possano essere apprezzati è quella della fedeltà della riproduzione. L'idea, peraltro, è perfettamente in linea con il criterio del $\pi \rho \varepsilon ́$ trov, che tanta parte abbiamo visto avere nella concezione di Plutarco, secondo quanto egli stesso dice in aud. poet. $18 \mathrm{~A}$ :

ciò che è intrinsecamente brutto non può diventare bello: quello che si apprezza, invece, è l'imitazione che sa ritrarre fedelmente un soggetto, cattivo o buono che sia; ma se, al con-

Cf. Pl., $R . \mathrm{X} 595 \mathrm{e}$ ss. dove l'attività del pittore è utilizzata come termine analogico per dimostrare la natura dell'attività del poeta, e Arist., $P o$. 1447a 19-28 dove è inclusa nel paragone anche la danza, e $1460 \mathrm{~b} 8$, in cui il poeta è esplicitamente paragonato al pittore o comunque ad un qualsiasi 'facitore di immagini'.

26 Cf. adulat. 58B (l'analogia, però, è ridotta alla prima metà), glor. Ath. 346F (Plutarco attribuisce il detto esplicitamente a Simonide di Ceo), quaest. conv. 748A (è confermata la paternità simonidea, ma alla pittura è sostituita la danza). Comunque, il paragone era piuttosto usuale e lo si ritrova anche in Rhet. ad Herenn. 4,28. Per un quadro completo sul problema della mimesi poetica in Plutarco, si vedano L. S. ScAzzocchio, Poética y Crítica Literaria en Plutarco, Montevideo, 1957, pp. 25 ss.; A. M. TagliasacchI, "Le teorie estetiche...", cit., pp. 83 s.; E. VAlGiGLIO, "Il tema della poesia...", cit., pp. 320 ss.; L. V DER STOCKT, "L'esperience esthétique de la mimésis selon Plutarque", QUCC, 36 (1990) 23-31; ID., op. cit., pp. 39-55 e, da ultimo, C. BRECHET, "Le audiendis poetis...", cit., pp. 220 ss. che riflette in modo particolare sulla relazione che intercorre tra mimesi e mythopoiia. Nel De audiendis poetis, per questo studioso, che segue e sviluppa gli argomenti di VAN DER STOCKT, Plutarco distinguerebbe tra il falso mitologico e la mimesi in sé e per sé, sì da intendere la poesia sia come arte del plattein sia come mimesi. In realtà, però, arriverebbe poi ad una effettiva 'conciliazione' tra i due momenti: la mimesi designerebbe la natura della poesia, la mythopoiia, invece, la fattura tecnica dei poemi, costituendo in pratica le due facce di una stessa medaglia. 
trario, rende belle le sembianze di una persona brutta, essa è venuta meno ai criteri della

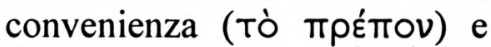
della somiglianza (Tò Eíkòs) (trad. di G. Pisani).

In quaest. conv. $673 \mathrm{C}$, però, si procede ancora oltre, perché insistendo sull'atteggiamento compiaciuto di chi fruisce dell'opera d'arte, anche quando è rappresentato il brutto, si indaga sulla particolare natura del godimento artistico e si scopre che il piacere deriva dal riconoscere $o$, meglio, dall' 'intuire' la bravura con cui l'opera è stata realizzata. L'uomo, infatti, è dotato di ragione e guarda con ammirazione le cose in cui si manifesta l'ingegnosità della mente. Tuttavia, per Plutarco il piacere estetico non sarebbe frutto di un'operazione 'intellettualistica' come lo è, ad esempio, per Aristotele ${ }^{28}$, bensì, piuttosto, effetto di un 'guizzo', di un qualcosa che si realizza in maniera naturale, spontanea, intuitiva, appunto, facendo riconoscere allo spettatore l'impronta della capacità ingegnosa dell'artista impressa nel prodotto artistico ${ }^{29}$.
Diversi i corollari del processo mimetico, che emergono in modo più o meno evidente anche nel giudizio critico su Aristofane e Menandro. Innanzi tutto, poiché l'arte è imitazione della vita, e la vita è multiforme e varia, col suo miscuglio di bene e male, è naturale che il prodotto artistico abbia come qualità intrinseca anche la 'varietà'30. E' questo un altro motivo di apprezzamento per Menandro, che sa adattare la sua espressione a vari personaggi (853D: $\pi \rho \circ \sigma \omega ́-$ mols mavtodamoĩs).

Quello che, invece, manca ad Aristofane, che pure ha nelle sue commedie tante differenze e varietà (853C-D: Sıaqo-

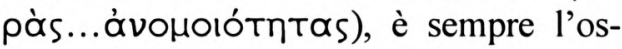
servanza del Tò $\pi \rho \varepsilon ́$ Trov, sicché la varietà stessa si traduce per lui in una vera

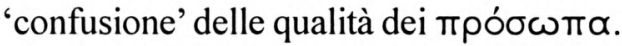

Sempre all'imitazione è legata, inoltre, l'esigenza di un linguaggio 'realistico', esigenza che risale fino ad Aristotele, ma che ebbe una sua sistemazione, per così dire, nella $\lambda{ }^{\prime} \xi$ Is ńӨıкń di Teofrasto, cui si è già accennato ${ }^{31}$. Plutarco, dunque,

Per lo Stagirita si tratta sostanzialmente della soddisfazione che si prova nel riconoscere gli oggetti rappresentati come oggetti già visti o nell'ammirarne la perfezione formale, il colore e così via. E' un'esperienza da ricondurre, dunque, al gusto della conoscenza.

Cf. L. VAN. DER StOckT, “L'experience esthétique...”, cit., pp. 28 ss.

Altrove questo stesso elemento estetico appare sviluppato autonomamente dalla realtà della vita e si ricollega ai soggetti dei miti che proprio per le $\mu \varepsilon \tau \alpha \beta \circ \lambda \alpha i$ rendono più efficace la funzione psicagogica esercitata sui lettori. Cf. A.M. TAGLIASACCHI, "Le teorie estetiche...", cit., pp. 97 s.; E. VALGIGLIO, "Il tema della poesia...", cit., pp. 323 s.; L. VAN DER STOCKT, op. cit., pp. $44 \mathrm{~s}$. 
non può non apprezzare il linguaggio corrente (853E: kolvoĩs...), familiare

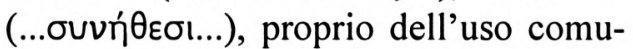
ne (...úTrò tìv Xpeíav), in cui si esprime Menandro, anche perché in esso appare notevolmente ridotto l'aspetto di quella purezza attica esageratamente perseguita da Aristofane ${ }^{32}$.

L'elemento più interessante di tutti, però, che caratterizza meglio la teoria estetica generale di Plutarco e che quindi ritorna anche nell'ambito della poesia comica, è la funzione propriamente didascalica che, raccogliendo il retaggio prearistotelico nonché stoico ${ }^{33}$, egli riconosce ad ogni forma di poesia.

Preoccupato com'è dell'uomo in quanto tale, delle sue possibilità di miglioramento e dei suoi pericoli di peggioramento, Plutarco considera fondamentale il 'messaggio' trasmesso dai componimenti poetici. Infatti, constatato che gli uomini in genere e in special modo i giovani non amano la severità delle speculazioni filosofiche, ma sono al contrario molto attratti dal diletto del mito e quindi della poesia, egli mira a ricercare il valore moralistico della stessa e arriva addirittura a strumentalizzare il piacere artistico, l'

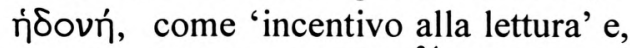
di qui, "molla alla morale, ${ }^{34}$.

È naturale, poi, che questo discorso valga in modo particolare per $\mathrm{i}$ testi drammatici, per la potenza di coinvolgimento che lo spettacolo teatrale esercita solitamente sugli spettatori ${ }^{35}$.

Tale prospettiva, d'altronde, anziché svalutare riesce anche a giustificare meglio la rappresentazione dei soggetti ignobili, dal momento che la commedia, mostrando la sconvenienza delle turpitudini, offre il suo insegnamento positivo e finisce per risultare non dannosa ${ }^{36}$. Arriviamo, così, ad un concetto di importanza

Cf. D. LANZA, "Lo spettacolo...", cit., p. 120; M. R. CAMmAROTA, "Estetica e critica letteraria...", cit., p. 104.

Al di fuori dello stretto ambito peripatetico, con la sola eccezione dei sofisti, era piuttosto diffusa la concezione della poesia come forma di insegnamento e Platone stesso, nonostante la forte avversione alla presenza della poesia nel suo stato ideale, riconosceva talora ai poemi un compito educativo (cf. Pl., Prt. 325e-326a e Lg. II 654a ). Dopo l'acuta critica aristotelica che aveva negato alla poesia qualsiasi intenzione educativa e aveva attribuito ad essa il piacere come fine precipuo, una ripresa dello schema concettuale tradizionale segnò la scuola stoica, recuperando come vero scopo della poesia gli ammaestramenti offerti al lettore, tesi a trasmettergli la verità e a incitarlo verso una buona condotta di vita. Per l'influenza stoica nelle tesi del De audiendis poetis si veda D. Babut., Plutarque et lo stö̈cisme, Paris, 1969, pp. 87-93.

Cf. E. Valgiglio, "Il tema della poesia...", cit., p. 329 e pp. 345 s.

Cf. D. LANZA, "Lo spettacolo...", cit., p.117; M. R. CAMMAROTA, "Estetica e critica letteraria...", cit., p. 95.

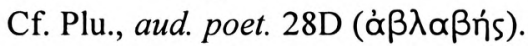


fondamentale, vale a dire l'innocuità della poesia faceta, che Plutarco assorbe allineandosi ancora una volta con chiara evidenza alla tradizione platonica ed aristotelica: sia Platone $\mathrm{e}^{37}$ che Aristotele ${ }^{38}$ accettavano, infatti, il ridicolo a patto che esso fosse 'innocuo'. Ma sono da rilevare differenze sostanziali: se per Platone si tratta di tutelare quanti sono oggetto di caricatura e per Aristotele di sostenere la funzione moderatrice del riso, Plutarco, piuttosto, dà l'impressione di preoccuparsi dell'effetto che la commedia può esercitare sull'animo dei suoi fruitori e, in modo particolare, dei giovani, passando appunto in modo più manifesto dalla semplice interpretazione moralistica ad una finalità propriamente didascalica ${ }^{39}$.

In conclusione, Plutarco è animato perennemente dalla sua vocazione pedagogica, per cui si preoccupa dell'utilità dei contenuti anziché dei pregi artistici in sé e per sé del prodotto poetico ed è senz'altro questo il motivo principale per cui rifiuta in blocco il teatro di
Aristofane preferendo ad esso quello di Menandro. Il fatto che Aristofane non sappia applicare i canoni artistici del

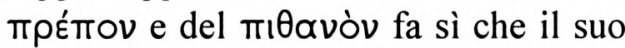
contenuto non sia 'utile', sicché viene a cadere il motivo primo di apprezzamento di un'opera d'arte secondo un criterio valutativo tipico dell'estetica antica.

Se nell'ottica di un'estetica moderna, infatti, il Cheronese sembra preoccuparsi solo di una qualità secondaria della poesia, quale era appunto la sua valenza educativa, secondo l'estetica antica, invece, proprio l'indagine del potere paideutico costituiva la valutazione del carattere primario ed essenziale della poesia $^{40}$. Vera poesia è per Plutarco, insomma, solo quella che insegna e, se diletta, diletta proprio per 'educare' ${ }^{41}$.

Il nostro opuscolo, tuttavia, offre uno spunto prezioso per cogliere un ulteriore aspetto del 'diletto'. Il terzo capitolo della Comparatio, infatti, dopo aver esaltato la Xápıs di Menandro, per cui si

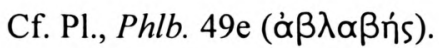

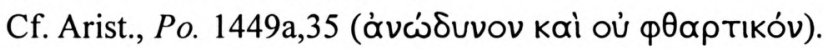

Una conferma di tale impressione è presente in un Col.1127A, dove Plutarco fa cenno esplicitamente all' 'utilità' che i poeti comici insieme con i poeti tragici tentano di offri-

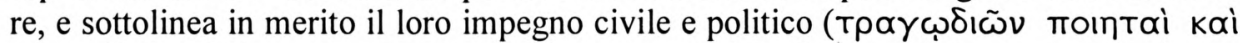

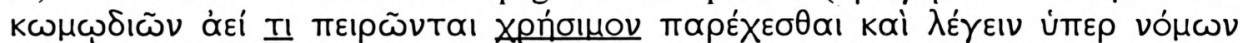

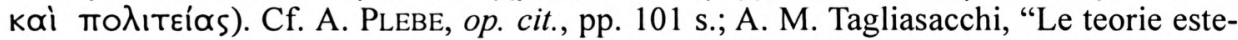
tiche...", cit., pp. 75 ss.; M. R. CAMMAROTA, "Estetica e critica letteraria...", cit., p. 93.

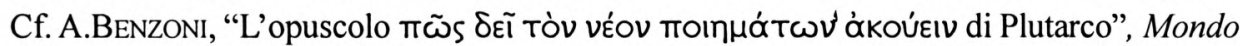
Classico, 3 (1933) 125-132, in part. pp. 125 ss. E. PANOFSKY, Idea, Firenze, 1952, p. 4.

Cf. L. S. ScAzZOCCHIO, op. cit., pp. 21 s.; A. M. TAGLIASACCHI, "Plutarco e la tragedia...", cit., p. 124; EAD. "Le teorie estetiche...", cit., pp. 75 ss.; M. R. CAMMAROTA, "Estetica e critica letteraria...", cit., p. 93. 
è sempre mostrato soddisfacente negli ambienti in cui è stato presentato, si chiude con una similitudine molto suggestiva che è tutta tesa ad esaltare la piacevolezza delle sue commedie:

in conclusione, come quando $\mathrm{i}$ pittori hanno gli occhi affaticati e si volgono ai colori dei fiori e dell'erba, per gli uomini dediti allo studio e operosi Menandro è il ristoro da quegli studi concentrati e intensi, quasi raccogliesse la mente in un prato ben fiorito e ombroso e pieno di brezze (854B-C).

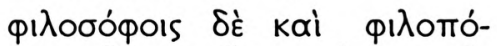

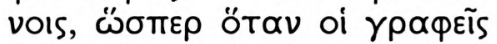

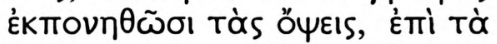

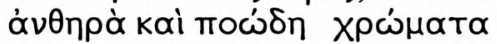

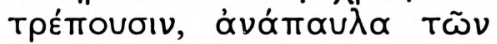

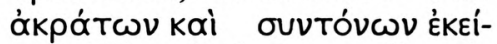

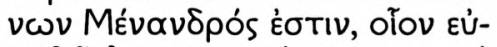

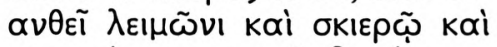

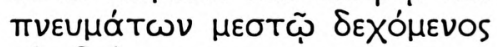

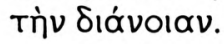

Si vuole evidentemente sottolineare l'amenità propria di opere che trattano fatti di vita quotidiana e che magari puntano l'attenzione sull'individuo nei suoi rapporti privati e nella sua evoluzione interiore, facendolo assurgere ad una dimensione universale, nell'aspirazione di cogliere un più profondo significato di umanità. Eppure, visto che nel contesto si allude a persone stanche, sembra si possa intuire anche un immediato riferimento all'espressione formale delle commedie e alla rassicurante sensazione che doveva procurare il riconoscerne il linguaggio piano e familiare. Si tratta di quel particolare diletto suscitato dal ritmo e dall'armonia dei versi, che operando un vero incantamento giova talora a mitigare le passioni e a ristabilire un sano equilibrio $^{42}$. D'altra parte non sarebbe nuovo per quanto concerne il poeta della véa, dal momento che altrove, con Euripide e Pindaro, egli è incluso nella triade dei poeti raccomandati per 'calmare' gli animi eccitati da una cattiva musica ${ }^{43}$.

Il Cheronese recupera insomma una sorta di funzione 'catartica' per la poesia menandrea, sia sul piano contenutistico che su quello più schiettamente formale. Detto questo, va da sé il confronto con il grande teorizzatore della 'catarsi' e si scopre quanto siano distanti le due posizioni. Se per Aristotele (che si riferisce specialmente alla tragedia) si tratta, infatti, di una 'purificazione' dalle passioni umane ottenuta per mezzo delle passioni stesse (destate e universalizzate dalla rappresentazione) e comunque sempre finalizzata al piacere artistico, per Plutarco si tratta invece di un'autentica moderazione di tutte le varie sfrenatezze e intemperanze, o semplicemente delle pene umane, con una finalità etico-educativa ${ }^{44}$. non letteraria in tutta l'opera di Plutarco, cf. L. VAN DER STOCKT, op. cit., pp. 140 ss. Cf. Plu., quaest. conv. 706D. 
La lettura della Comparatio, pertan- che attrae, diletta e, al tempo stesso, preto, ci aiuta a cogliere finalmente in tutta para gli animi ad accogliere i contenuti di la sua pregnanza la nota pagina relativa saggezza, e l'altro, invece, all'elemento alla genesi della commedia ${ }^{45}$ : il $\phi 1 \lambda \circ-$ più puramente razionale, che dà nutrimen-

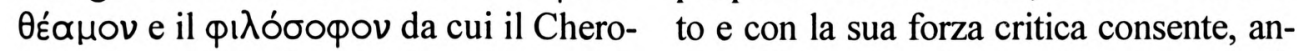
nese fa nascere il genere comico, corri- che nella commedia, una vera e propria spondono l'uno all'elemento spettacolare, forma di 'prefilosofia, ${ }^{, 46}$.

45 Plu., quaest. conv. 673B.

46 Cf. A. Plebe, op. cit., pp. 102 ss.; A. M. TAgliasaCchi, "Le teorie estetiche...", cit., pp. 72 ss. 